Pacific

Journal of

Mathematics

THE JUMPING PHENOMENON OF HODGE NUMBERS

XUANMing Ye

Volume $235 \quad$ No. 2

April 2008 


\title{
THE JUMPING PHENOMENON OF HODGE NUMBERS
}

\author{
XUANMing Ye
}

\begin{abstract}
Let $X$ be a compact complex manifold, and let $\pi: \mathscr{L} \rightarrow B$ be a small deformation of $X$. The dimensions of the Dolbeault cohomology groups $H^{q}\left(X_{t}, \Omega_{X_{t}}^{p}\right)$ may vary under this deformation. In this paper, we will study the deformation obstructions of a $(p, q)$-class in the central fiber $X$. In particular, we obtain an explicit formula for the obstructions and apply this formula to the study of small deformations of the Iwasawa manifold.
\end{abstract}

\section{Introduction}

Let $X$ be a compact complex manifold and $\pi: \mathscr{X} \rightarrow B$ be a family of complex manifolds such that $\pi^{-1}(0)=X$. Let $X_{t}=\pi^{-1}(t)$ denote the fiber of $\pi$ over the point $t \in B$. We denote by $\mathcal{O}_{X}$ and $\Omega_{X}^{p}$ the sheaf of germs of holomorphic functions of $X$ and holomorphic $p$-forms of $X$ respectively. Recall $h^{p, q}=\operatorname{dim}_{\mathbb{C}} H^{q}\left(X, \Omega_{X}^{p}\right)$ (dim will denote complex dimension throughout) and $P_{l}=\operatorname{dim} H^{0}\left(X,\left(\Omega_{X}^{m}\right)^{\otimes l}\right.$ ) where $m=\operatorname{dim} X$. S. Iitaka proposed a problem whether $h^{p, q}$ and $P_{l}$ are deformation invariants [Iitaka 1972]. This problem was solved by Iku Nakamura in [1975], and actually he gave us some examples of small deformations of complex parallelizable manifold (by a complex parallelizable manifold we mean a compact complex manifold with a trivial holomorphic tangent bundle) such that the Hodge numbers of the fiber of the family jump in these deformations.

In this paper, we will study such phenomena from the viewpoint of obstruction theory. More precisely, for a certain small deformation $\mathscr{X}$ of $X$ parametrized by a basis $B$ and a certain class $[\alpha]$ of the Dolbeault cohomology group $H^{q}\left(X, \Omega_{X}^{p}\right)$, we will try to find out the obstruction to extend it to an element of the relative Dolbeault cohomology group $H^{q}\left(\mathscr{X}, \Omega_{\mathscr{X} / B}^{p}\right)$. We will call those elements which have nontrivial obstruction the obstructed elements. And then we will see that these elements will play an important role when we study the jumping phenomenon of Hodge numbers, because we will see that the existence of the obstructed elements is a necessary and sufficient condition for the variation of the Hodge diamond.

MSC2000: 20G10, 13D10, 32G05.

Keywords: Hodge number, Deformation, Obstruction, Kodaira Spencer Class.

The research was partially supported by China-France-Russian mathematics collaboration grant 34000-3275100, from Sun Yat-Sen University. 
In Section 2 we will summarize the results of Grauert's Direct Image Theorems from which we get a "model" of the jumping phenomenon of Hodge numbers and then we will try to explain why we need to consider the obstructed elements. The relation between the jumping phenomenon of Hodge numbers and the obstructed elements is the following.

Theorem 2.11. Let the compact complex manifold $X$ be the central fiber of a small deformation $\pi: \mathscr{X} \rightarrow B$ of $X$. Now we consider $\operatorname{dim} H^{q}\left(X_{t}, \Omega_{X_{t}}^{p}\right)$ as a function of $t \in B$. It jumps at $t=0$ if and only if there exists an element $\alpha$ either in $H^{q}\left(X, \Omega_{X}^{p}\right)$ or in $H^{q-1}\left(X, \Omega_{X}^{p}\right)$ and a minimal natural number $n \geq 1$ such that the $n$-th order obstruction

$$
o_{n, n-1}(\alpha) \neq 0 .
$$

In Section 3 we will get a formula for the obstruction to the extension we mentioned above.

Theorem 3.5. Let $\pi: \mathscr{X} \rightarrow B$ be a deformation of $\pi^{-1}(0)=X$, where $X$ is a compact complex manifold. Let $\pi_{n}: X_{n} \rightarrow B_{n}$ be the $n$-th order deformation of $X$. For arbitrary $[\alpha]$ that belongs to $H^{q}\left(X, \Omega_{X}^{p}\right)$, suppose we can extend $[\alpha]$ to order $n-1$ in $H^{q}\left(X_{n-1}, \Omega_{X_{n-1} / B_{n-1}}^{p}\right)$. Denote such an element by $\left[\alpha_{n-1}\right]$. The obstruction of the extension of $[\alpha]$ to $n$-th order is given by

$$
o_{n, n-1}(\alpha)=d_{X_{n-1} / B_{n-1}} \circ \kappa_{n}\left\llcorner\left(\alpha_{n-1}\right)+\kappa_{n}\left\llcorner\circ d_{X_{n-1} / B_{n-1}}\left(\alpha_{n-1}\right),\right.\right.
$$

where $\kappa_{n}$ is the $n$-th order Kodaira-Spencer class and $d_{X_{n-1} / B_{n-1}}$ is the relative differential operator of $(n-1)$-th order deformation.

In Section 4 we will use this formula to study carefully the example given by Iku Nakamura, that is, the small deformation of the Iwasawa manifold, and to discuss some phenomena.

\section{Grauert's Direct Image Theorems and deformation theory}

In this section, let us first review some general results of deformation theory. Let $X$ be a compact complex manifold. $X$ has an underlying differential structure, but given this fixed differential structure there may be many different complex structures on $X$. In particular, there might be a range of complex structures on $X$ varying in an analytic manner. This is the object that we will study (see [Voisin 2002]).

Definition 2.1. A deformation of $X$ consists of a smooth proper morphism $\pi: \mathscr{L} \rightarrow$ $B$, where $\mathscr{X}$ and $B$ are connected complex spaces, and an isomorphism $X \cong \pi^{-1}(0)$, where $0 \in B$ is a distinguished point. We call $\mathscr{X} \rightarrow B$ a family of complex manifolds.

Although $B$ is not necessarily a manifold, and can be singular, reducible, or nonreduced (for example, $B=\operatorname{Spec} \mathbb{C}[\varepsilon] /\left(\varepsilon^{2}\right)$ ), since the problem we are going 
to study is the phenomenon of the jumping of the Dolbeault cohomology, we may assume that $\mathscr{L}$ and $B$ are complex manifolds.

In order to study the jumping of the Dolbeault cohomology, we need the following important theorem (one of the Grauert's Direct Image Theorems, see [Bell and Narasimhan 1990]).

Theorem 2.2. Let $X, Y$ be complex spaces, $\pi: X \rightarrow Y$ a proper holomorphic map. Suppose that $Y$ is Stein, and let $\mathscr{F}$ be a coherent analytic sheaf on $X$. Let $Y_{0}$ be a relatively compact open set in $Y$. Then, there is an integer $N>0$ such that the following hold.

(i) There exists a complex

$$
\mathscr{E}: \cdots \rightarrow \mathscr{E}^{-1} \rightarrow \mathscr{E}^{0} \rightarrow \cdots \rightarrow \mathscr{E}^{N} \rightarrow 0
$$

of finitely generated locally free $\mathrm{O}_{Y_{0}}$-modules on $Y_{0}$ such that for any Stein open set $W \subset Y_{0}$, we have

$$
H^{q}\left(\Gamma\left(W, \mathscr{E}^{\cdot}\right)\right) \simeq \Gamma\left(W, R^{q} \pi_{*}(\mathscr{F})\right) \simeq H^{q}\left(\pi^{-1}(W), \mathscr{F}\right) \quad \forall q \in \mathbb{Z} .
$$

(ii) (Base Change Theorem). Assume, in addition, that $\mathscr{F}$ is $\pi$-flat, that is, for all $x \in X$ the stalk $\mathscr{F}_{x}$ is flat as a module over $\mathbb{O}_{Y, \pi(x)}$. Then, there exists a complex

$$
\mathscr{E}: \cdots \rightarrow \mathscr{E}^{-1} \rightarrow \mathscr{E}^{0} \rightarrow \cdots \rightarrow \mathscr{E}^{N} \rightarrow 0
$$

of finitely generated locally free $\mathscr{O}_{Y_{0}}$-sheaves $\mathscr{E}^{p}$ with the following property:

Let $S$ be a Stein space and $f: S \rightarrow Y$ a holomorphic map. Let $X^{\prime}=X \times_{Y} S$ and $f^{\prime}: X^{\prime} \rightarrow X$ and $\pi^{\prime}: X^{\prime} \rightarrow S$ be the two projections. Then, if $T$ is an open Stein subset of $f^{-1}\left(Y_{0}\right)$, we have, for all $q \in \mathbb{Z}$,

$$
H^{q}\left(\Gamma\left(T, f^{*}\left(\mathscr{E}^{*}\right)\right)\right) \simeq \Gamma\left(T, R^{q} \pi_{*}^{\prime}\left(\mathscr{F}^{\prime}\right)\right) \simeq H^{q}\left(\pi^{\prime-1}(T), \mathscr{F}^{\prime}\right)
$$

where $\mathscr{F}^{\prime}=\left(f^{\prime}\right)^{*}(\mathscr{F})$.

Let $X, Y$ be complex spaces and $\pi: X \rightarrow Y$ a proper map. Let $\mathscr{F}$ be a $\pi$ flat coherent sheaf on $X$. For $y \in Y$, denote by $\mu_{y}$ the $O_{Y}$-sheaf of germs of holomorphic functions "vanishing at y", that is, the stalk of $\mu_{y}$ at $\mathrm{y}$ is the maximal ideal of $O_{Y, y}$ and the stalks at $t \neq y$ are $\mathscr{O}_{Y, t}$. Let $\mathscr{F}(y)$ be the analytic restriction of $\mathscr{F}$ to $\pi^{-1}(y)$, that is, $\mathscr{F}_{\mathcal{F}} \otimes_{\mathscr{O}_{Y}}\left(\mathscr{O}_{Y} / M_{y}\right)$. Since we just need to study the local properties, we may assume that there is a complex

$$
\mathscr{E}^{\cdot}: 0 \stackrel{d}{\longrightarrow} \mathcal{O}_{Y}^{P_{0}} \stackrel{d^{0}}{\longrightarrow} \mathrm{O}_{Y}^{P_{1}} \stackrel{d^{1}}{\longrightarrow} \cdots \stackrel{d^{N-1}}{\longrightarrow} \mathrm{O}_{Y}^{P_{N}} \stackrel{d^{N}}{\longrightarrow} 0
$$

in view of the base change property in Theorem 2.2 part (ii). In particular, if $y \in Y$, we have

$$
H^{q}\left(\pi^{-1}(y), \mathscr{F}(y)\right) \simeq H^{q}\left(\mathscr{E} \cdot \otimes\left(\mathcal{O}_{Y} / M_{y}\right)\right)
$$


Apply what we discussed above to our case $\pi: \mathscr{L} \rightarrow B$, we get the following. There is a complex of vector bundles on the basis $B$, whose cohomology groups at the point identify to the cohomology groups of the fiber $X_{t}$ with values in the considered vector bundles on $\mathscr{X}$, restricted to $X_{t}$. Therefore, for arbitrary $p$, there exists a complex of vector bundles $\left(E^{*}, d^{*}\right)$ such that for any $t \in B$,

$$
H^{q}\left(X_{t}, \Omega_{X_{t}}^{p}\right)=H^{q}\left(E_{t}^{\cdot}\right)=\operatorname{Ker}\left(d^{q}\right) / \operatorname{Im}\left(d^{q-1}\right) .
$$

Via a local trivialization of the bundle $E^{i}$, the differential of the complex $E$ is represented by matrices with holomorphic coefficients, and follows from the lower semicontinuity of the rank of a matrix with variable coefficients, it is easy to check that the functions $\operatorname{dim} \operatorname{Ker}\left(d^{q}\right)$ and $\left(-\operatorname{dim} \operatorname{Im}\left(d^{q}\right)\right)$ are upper semicontinuous on $B$. Therefore the function $\operatorname{dim} H^{q}\left(E_{t}^{\cdot}\right)$ is also upper semicontinuous. It seems that either the increasing of $\operatorname{dim} \operatorname{Im}\left(d^{q-1}\right)$ or the decreasing of $\operatorname{dim} \operatorname{Ker}\left(d^{q}\right)$ will cause the jumping of $\operatorname{dim} H^{q}\left(E_{t}^{\cdot}\right)$; however, because of the following exact sequence

$$
0 \rightarrow \operatorname{Ker}\left(d^{q}\right)_{t} \rightarrow E_{t}^{q} \rightarrow \operatorname{Im}\left(d^{q}\right)_{t} \rightarrow 0 \quad \forall t
$$

implying the variation of $\left(-\operatorname{dim} \operatorname{Im}\left(d^{q}\right)\right)$ is exactly the variation of $\operatorname{dim} \operatorname{Ker}\left(d^{q}\right)$, we just need to consider the variation of $\operatorname{dim} \operatorname{Ker}\left(d^{q}\right)$ for all $q$.

In order to study the variation of $\operatorname{dim} \operatorname{Ker}\left(d^{q}\right)$, we need to consider the following problem. Let $\alpha$ be an element of $\operatorname{Ker}\left(d^{q}\right)$ at $t=0$, we try to find out the obstruction to extend $\alpha$ to an element which belongs to $\operatorname{Ker}\left(d^{q}\right)$ in a neighborhood of 0 . Such kind of extension can be studied order by order. Let $\mathscr{E}_{0}^{q}$ be the stalk of the associated sheaf of $E^{q}$ at 0 . Let $m_{0}$ be the maximal ideal of $O_{B, 0}$. For arbitrary positive integer $n$, since $d^{q}$ can be represented by matrices with holomorphic coefficients, it is not difficult to check

$$
d^{q}\left(\mathscr{E}_{0}^{q} \otimes_{\mathscr{O}_{B, 0}} m_{0}^{n}\right) \subset \mathscr{E}_{0}^{q+1} \otimes_{\mathbb{O}_{B, 0}} m_{0}^{n} .
$$

Therefore the complex of the vector bundles $\left(E^{\cdot}, d^{*}\right)$ induces the complex

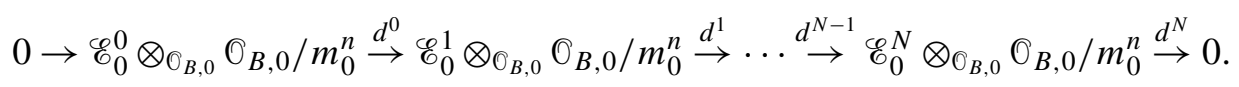

Definition 2.3. Those elements of $H^{\cdot}\left(E_{0}^{\cdot}\right)$ which cannot be extended are called the first class obstructed elements.

Next, we will show the obstructions of the extension we mentioned above. For simplicity, we may assume that $\operatorname{dim} B=1$ and suppose $\alpha$ can be extended to an element $\alpha_{n-1}$ such that $j_{0}^{n-1}\left(d^{q}\left(\alpha_{n-1}\right)\right)(t)=0$, where $j_{0}^{n-1}\left(d^{q}\left(\alpha_{n-1}\right)\right)(t)$ is the $(n-1)$-jet of $d^{q}\left(\alpha_{n-1}\right)$ at 0 . Then $\alpha_{n-1}$ can be considered as the $(n-1)$-order extension of $\alpha$. 
Remark 2.4. Suppose that $f: \mathbb{R} \rightarrow \mathbb{R}$ is a real-valued function having at least $k+1$ derivatives in a neighborhood $U$ of the point $x_{0}$. Then by Taylor's Theorem,

$$
f(x)=f\left(x_{0}\right)+f^{\prime}\left(x_{0}\right)\left(x-x_{0}\right)+\cdots+\frac{f^{k}\left(x_{0}\right)}{k !}\left(x-x_{0}\right)^{k}+\frac{R_{k+1}(x)}{(k+1) !}\left(x-x_{0}\right)^{k+1}
$$

where

$$
\left|R_{k+1}(x)\right| \leq \sup _{x \in U}\left|f^{k+1}(x)\right| .
$$

Then the $k$-jet of $f$ at the point $x_{0}$ is defined to be the polynomial

$$
j_{x_{0}}^{k}(f)(z)=f\left(x_{0}\right)+f^{\prime}\left(x_{0}\right) z+\cdots+\frac{f^{k}\left(x_{0}\right)}{k !} z^{k} .
$$

Suppose that $M$ is a smooth manifold containing a point $p$. We shall define the jets of curves through $p$, by which we henceforth mean smooth functions $f: \mathbb{R} \rightarrow$ $M$ such that $f(0)=p$. Define an equivalence relation $\mathrm{Eq}_{p}^{k}$ as follows. Let $f$ and $g$ be a pair of curves through $p$. We will then say that $f$ and $g$ are equivalent to order $k$ at $p$ if there is a neighborhood $U$ of $p$ such that every smooth function $\varphi: U \rightarrow \mathbb{R}$ satisfies $j_{0}^{k}(\varphi \circ f)=j_{0}^{k}(\varphi \circ g)$. Note that these jets are well-defined since the composite functions $\varphi \circ f$ and $\varphi \circ g$ are just mappings from the real line to itself. This equivalence relation is sometimes called that of $k$-th order contact between curves at $p$. The $k$-jet of a curve $f$ through $p$ is defined to be the equivalence class of $f$, and is denoted by $j_{p}^{k} f$.

We are now prepared to define the jet of a function from a manifold to a manifold. Suppose that $M$ and $N$ are two smooth manifolds. Let $p$ be a point of $M$. Consider the space $C_{p}^{\infty}(M, N)$ consisting of smooth maps $f: M \rightarrow N$ defined in some neighborhood of $p$. We define an equivalence relation $\mathrm{Eq}_{p}^{k}$ on $C_{p}^{\infty}(M, N)$ as follows. Two maps $f$ and $g$ are said to be equivalent if, for every curve $\gamma$ through $p$ (recall that by our conventions this is a mapping such that $\gamma(0)=p$ ), we have $j_{0}^{k}(f \circ \gamma)=j_{0}^{k}(g \circ \gamma)$ on some neighborhood of 0 . We define the $k$-jet of $f$ at $p$, $j_{p}^{k} f$, to be the equivalence class of $f$ modulo $E q_{p}^{k}$.

In our case, via a local trivialization of the bundles $E^{q+1}, j_{0}^{n-1}\left(d^{q}\left(\alpha_{n-1}\right)\right)(t)$ is represented by the $(n-1)$-jets of the coefficients of $d^{q}\left(\alpha_{n-1}\right)$.

Define a map

$$
\begin{gathered}
o_{n}^{q}: H^{q}\left(\mathscr{E}_{0} \otimes_{\mathcal{O}_{B, 0}} \mathbb{O}_{B, 0} / m_{0}^{n}\right) \rightarrow H^{q+1}\left(E_{0}^{\cdot}\right) \\
{\left[\alpha_{n-1}\right] \longmapsto\left[j_{0}^{n}\left(d^{q}\left(\alpha_{n-1}\right)\right)(t) / t^{n}\right] .}
\end{gathered}
$$

First, we need to check $o_{n}^{q}$ is well defined. So we need to show that

$$
\left[j_{0}^{n}\left(d^{q}\left(\alpha_{n-1}\right)\right)(t) / t^{n}\right]
$$


is $d^{q+1}$-closed. Via a local trivialization of the bundles $E^{i}$, the differentials of the complex $E^{*}$ are represented by matrices with holomorphic coefficients, and from the lower semicontinuity of the rank of a matrix with variable coefficients, we may assume that there always exist $\left(\sigma_{1}^{q+1}, \ldots, \sigma_{l}^{q+1}\right)$ which are sections of $E^{q+1}$ such that

$$
\left(\left.\sigma_{1}^{q+1}\right|_{t=0}, \ldots,\left.\sigma_{l}^{q+1}\right|_{t=0}\right)
$$

form a basis of

$$
\operatorname{Ker}\left(d^{q+1}: E_{0}^{q+1} \rightarrow E_{0}^{q+2}\right) \quad \text { and } \quad \operatorname{Ker}\left(d^{q+1}: E^{q+1} \rightarrow E^{q+2}\right) \subset \operatorname{Span}\left\{\sigma_{j}^{q+1}\right\} .
$$

So we can write $d^{q}\left(\alpha_{n-1}\right)=\sum_{j} f_{j} \sigma_{j}^{q+1}$.

Since $j_{0}^{n-1}\left(d^{q}\left(\alpha_{n-1}\right)\right)(t)=0$, we have $f_{j}(0)=0$ and $\frac{\partial^{i} f}{\partial t^{i}}(0)=0, i=1, \ldots, n-1$.

$$
\begin{aligned}
& \left.\frac{\partial^{n}}{\partial t^{n}}\left(d^{q}\left(\alpha_{n-1}\right)\right)\right|_{t=0}= \\
& \qquad\left.\sum_{j} \frac{\partial^{n} f_{j}}{\partial t^{n}} \sigma_{j}^{q+1}\right|_{t=0}+\cdots+\left.\sum_{j} f_{j} \frac{\partial^{n}}{\partial t^{n}}\left(\sigma_{j}^{q+1}\right)\right|_{t=0}=\left.\sum_{j} \frac{\partial^{n} f_{j}}{\partial t^{n}} \sigma_{j}^{q+1}\right|_{t=0} .
\end{aligned}
$$

Therefore

$$
d^{q+1}\left(\left.\frac{\partial^{n}}{\partial t^{n}}\left(d^{q}\left(\alpha_{n-1}\right)\right)\right|_{t=0}\right)=d^{q+1}\left(\left.\sum_{j} \frac{\partial^{n} f_{j}}{\partial t^{n}} \sigma_{j}^{q+1}\right|_{t=0}\right)=0,
$$

which means $\left.\frac{\partial^{n}}{\partial t^{n}}\left(d^{q}\left(\alpha_{n-1}\right)\right)\right|_{t=0}$ is $d^{q+1}$-closed.

Next we are going to show that the equivalent class of $\left.\frac{\partial^{n}}{\partial t^{n}}\left(d^{q}\left(\alpha_{n-1}\right)\right)\right|_{t=0}$ in $H^{q+1}\left(E_{0}\right)$ depends only on $j_{0}^{n-1}\left(\alpha_{n-1}\right)(t)$. Let $\left(\sigma_{1}^{q}, \ldots, \sigma_{k}^{q}\right)$ be a basis of $E^{q}$, we only need to show that if $j_{0}^{n-1}\left(\alpha_{n-1}\right)(t)=0$, then $\left.\frac{\partial^{n}}{\partial t^{n}}\left(d^{q}\left(\alpha_{n-1}\right)\right)\right|_{t=0}$ belongs to $\operatorname{Im}\left(d^{q}: E^{q} \rightarrow E^{q+1}\right)$. Indeed, we can write $\alpha_{n-1}=\sum_{j} f_{j} \sigma_{j}^{q}$ while $f_{j}(0)=0$, $\frac{\partial^{i} f}{\partial t^{i}}=0, i=1, \ldots, n-1$, then

$$
\begin{aligned}
\frac{\partial^{n}}{\partial t^{n}}\left(d^{q}\left(\alpha_{n-1}\right)\right)=\frac{\partial^{n}}{\partial t^{n}}\left(\sum_{i} f_{i} d^{q}\left(\sigma_{i}^{q}\right)\right) & \\
& =\sum_{i} \frac{\partial^{n} f_{i}}{\partial t^{n}} d^{q}\left(\sigma_{i}^{q}\right)+\cdots+\sum_{i} f_{i} \frac{\partial^{n}}{\partial t^{n}}\left(d^{q}\left(\sigma_{i}^{q}\right)\right) .
\end{aligned}
$$

Therefore,

$$
\left.\frac{\partial^{n}}{\partial t^{n}}\left(d^{q}\left(\alpha_{n-1}\right)\right)\right|_{t=0}=\left.\sum_{i} \frac{\partial^{n} f_{i}}{\partial t^{n}} d^{q}\left(\sigma_{i}^{q}\right)\right|_{t=0},
$$

which belongs to $\operatorname{Im}\left(d^{q}: E^{q} \rightarrow E^{q+1}\right)$. 
Last, we are going to show that the equivalent class of $\left.\frac{\partial^{n}}{\partial t^{n}}\left(d^{q}\left(\alpha_{n-1}\right)\right)\right|_{t=0}$ in $H^{q+1}\left(E_{0}\right)$ depends only on the equivalent class of $\alpha_{n-1}$ in $H^{q}\left(\mathscr{E}_{0} \otimes_{\mathcal{O}_{B, 0}} \widehat{O}_{B, 0} / m_{0}^{n}\right)$. Actually, we only need to show that if

$$
\left[\alpha_{n-1}\right] \in \operatorname{Im}\left(d^{q-1}: \mathscr{E}_{0}^{q-1} \otimes_{\mathscr{C}_{B, 0}} \mathcal{O}_{B, 0} / m_{0}^{n} \rightarrow \mathscr{E}_{0}^{q} \otimes_{\mathscr{O}_{B, 0}} \mathcal{O}_{B, 0} / m_{0}^{n}\right),
$$

we will have that $\left.\frac{\partial^{n}}{\partial t^{n}}\left(d^{q}\left(\alpha_{n-1}\right)\right)\right|_{t=0}$ belongs to $\operatorname{Im}\left(d^{q}: E^{q} \rightarrow E^{q+1}\right)$. In fact, let $\alpha_{n-1}^{\prime}=d^{q-1}\left(\sum_{j} f_{j} \sigma_{j}^{q-1}\right)$ such that $j_{0}^{n-1}\left(\alpha_{n-1}^{\prime}\right)(t)=j_{0}^{n-1}\left(\alpha_{n-1}\right)(t)$. From the discussion above, we have

$$
\left.\frac{\partial^{n}}{\partial t^{n}}\left(d^{q}\left(\alpha_{n-1}\right)\right)\right|_{t=0}=\left.\frac{\partial^{n}}{\partial t^{n}}\left(d^{q}\left(\alpha_{n-1}^{\prime}\right)\right)\right|_{t=0}=\frac{\partial^{n}}{\partial t^{n}}\left(d^{q}\left(d^{q-1}\left(\sum_{j} f_{j} \sigma_{j}^{q-1}\right)\right)\right)=0
$$

in $H^{q}\left(E^{\cdot}\right)$.

Remark 2.5. It seems that $j_{0}^{n}\left(d^{q}\left(\alpha_{n-1}\right)\right)(t) / t^{n}$ depends on the connection of $E^{q+1}$. But, by using an induction argument, it is not difficult to prove that if $j_{0}^{i}\left(d^{q}\left(\alpha_{n-1}\right)\right)(t)=0$ for all $i<n$, then $j_{0}^{n}\left(d^{q}\left(\alpha_{n-1}\right)\right)(t)$ is independent of the choice of the connection of $E^{q+1}$.

There is a natural map

$$
\rho_{i}^{q}: H^{q}\left(E_{0}^{\cdot}\right) \rightarrow H^{q}\left(\mathscr{E}_{0} \otimes_{\mathscr{O}_{B, 0}} \mathscr{O}_{B, 0} / m_{0}^{i+1}\right)
$$

given by $[\sigma] \longmapsto\left[t^{i} \sigma\right]$ for all $[\sigma] \in H^{q}\left(E_{0}^{\cdot}\right)$. For all $i \leq n$, denote by $o_{n, i}^{q}$ the map

$$
\rho_{i}^{q+1} \circ o_{n}^{q}: H^{q}\left(\mathscr{E}_{0} \otimes_{\mathbb{O}_{B, 0}} \mathcal{O}_{B, 0} / m_{0}^{n}\right) \rightarrow H^{q+1}\left(\mathscr{\mathscr { C }}_{0} \otimes_{\mathbb{O}_{B, 0}} \mathscr{O}_{B, 0} / m_{0}^{i+1}\right) .
$$

Next we will show that, for arbitrary $i$ with $0<i \leq n, \alpha_{n-1}$ can be extended to $\alpha_{n}$, the $n$-th order extension of $\alpha$, so that $j_{0}^{i-1}\left(\alpha_{n}-\alpha_{n-1}\right)(t)=0$ if and only if $o_{n, n-i}^{q}\left(\left[\alpha_{n-1}\right]\right)$ is trivial. For necessity, $j_{0}^{i}\left(\alpha_{n}-\alpha_{n-1}\right)(t) / t^{i}$ is supposed to be the preimage of $o_{n, n-i}^{q}\left(\left[\alpha_{n-1}\right]\right)$, so $o_{n, n-i}^{q}\left(\left[\alpha_{n-1}\right]\right)$ is trivial. Therefore we just need to check whether it is sufficient. In fact, if $o_{n, n-i}^{q}\left(\left[\alpha_{n-1}\right]\right)$ is trivial, then there exists a section $\beta$ of $\mathscr{E}_{0} \otimes_{\mathbb{O}_{B, 0}} \mathcal{O}_{B, 0} / m_{0}^{n-i+1}$ such that $d^{q}(\beta)=o_{n, n-i}^{q}\left(\left[\alpha_{n-1}\right]\right)$. Then it is not difficult to check that $\alpha_{n-1}-t^{i} \tilde{\beta}$ is an $n$-th order extension of $\alpha$ that we need, where $\tilde{\beta}$ is an element in the cohomology class of $\beta$. Therefore we have the following proposition.

Proposition 2.6. Let $\alpha_{n-1}$ be an $(n-1)$-th order extension of $\alpha$. For arbitrary $i$, $0<i \leq n, \alpha_{n-1}$ can be extended to $\alpha_{n}$ which is an $n$-th order extension of $\alpha$ such that $j_{0}^{i-1}\left(\alpha_{n}-\alpha_{n-1}\right)(t)=0$ if and only if $o_{n, n-i}^{q}\left(\left[\alpha_{n-1}\right]\right)=0$.

In the following, we will show that the obstructions $o_{n}^{q}\left(\left[\alpha_{n-1}\right]\right)$ also play an important role when we consider the jumping of $\operatorname{dim} \operatorname{Im}\left(d^{q}\right)$. Note that $\operatorname{dim} \operatorname{Im}\left(d^{q}\right)$ jumps if and only if there exist a section $\beta$ of $\operatorname{Ker}\left(d^{q+1}\right)$ such that $\beta_{0}$ is not exact while $\beta_{t}$ is exact for $t \neq 0$. 
Definition 2.7. Those nontrivial elements of $H^{\cdot}\left(E_{0}^{*}\right)$ that can always be extended to a section which is only exact at $t \neq 0$ are called the second class obstructed elements.

Note that if $\alpha$ is exact at $t=0$, it can be extended to an element which is exact at every point. So the definition above does not depend on the element of a fixed equivalent class.

Proposition 2.8. Let $[\beta]$ be an nontrivial element of $H^{q+1}\left(E_{0}^{\cdot}\right)$. Then $[\beta]$ is a second class obstructed element if and only if there exist $n \geq 0$ and $\alpha_{n-1}$ in $H^{q}\left(\mathscr{E}_{0} \otimes_{\mathscr{O}_{B, 0}} \bigcirc_{B, 0} / m_{0}^{n}\right)$ such that $o_{n}^{q}\left(\left[\alpha_{n-1}\right]\right)=[\beta]$.

Proof. If $o_{n}^{q}\left(\left[\alpha_{n-1}\right]\right)=[\beta]$, then $j_{0}^{n}\left(d^{q}\left(\alpha_{n-1}\right)\right)(t) / t^{n}$ is the extension we need. On the contrary, if $[\beta]$ is a second class obstructed element. There exist $\tilde{\beta}$ such that $\tilde{\beta}_{t}, t \neq 0$ is exact. Then the preimage $\tilde{\beta}^{\prime}$ of $\tilde{\beta}$ is a meromorphic section which has a pole at $t=0$. Let $n$ be the degree of $\tilde{\beta}^{\prime}$. Then let $\alpha_{n-1}=t^{n} \tilde{\beta}^{\prime}$. It is easy to check that $o_{n}^{q}\left(\left[\alpha_{n-1}\right]\right)=[\beta]$.

Proposition 2.9. Let $\alpha_{n-1}$ be an element of $H^{q}\left(\mathscr{E}_{0} \otimes_{\mathbb{O}_{B, 0}} \mathscr{O}_{B, 0} / m_{0}^{n}\right)$ such that $o_{n}^{q}\left(\left[\alpha_{n-1}\right]\right) \neq 0$. Then there exist $n^{\prime} \leq n$ and $\alpha^{\prime} \in H^{q}\left(\mathscr{E}_{0} \otimes_{\mathscr{O}_{B, 0}} \mathscr{O}_{B, 0} / m_{0}^{n^{\prime}}\right)$ such that

$$
\rho_{n^{\prime}-1}^{q+1} \circ o_{n}^{q}\left(\left[\alpha_{n-1}\right]\right)=o_{n^{\prime}, n^{\prime}-1}^{q}\left(\left[\alpha^{\prime}\right]\right) \neq 0 .
$$

Proof. If $o_{n, n-1}^{q}\left(\left[\alpha_{n-1}\right]\right) \neq 0$, then $n^{\prime}=n$ and $\alpha^{\prime}=\alpha_{n-1}$. Otherwise, there exists $\alpha_{1}^{\prime}$ such that $d^{q}\left(\alpha_{1}^{\prime}\right)=\rho_{n-1}^{q+1} \circ o_{n}^{q}\left(\left[\alpha_{n-1}\right]\right)$. Note that

$$
o_{n-1, n-2}^{q}\left(\left[\alpha_{1}^{\prime}\right]\right)=\rho_{n-2}^{q+1} \circ o_{n}^{q}\left(\left[\alpha_{n-1}\right]\right)=o_{n, n-2}^{q}\left(\left[\alpha_{n-1}\right]\right) .
$$

If we go on step by step as above, we can always get the $n^{\prime}$ and $\alpha^{\prime}$, for at least one of the $o_{n, i}^{q}\left(\left[\alpha_{n-1}\right]\right)$ is nontrivial.

This proposition tells us that although $o_{n}^{q}\left(\left[\alpha_{n-1}\right]\right) \neq 0$ does not mean that

$$
o_{n, n-1}^{q}\left(\left[\alpha_{n-1}\right]\right) \neq 0,
$$

we can always find $\alpha^{\prime}$ so that $o_{n}^{q}\left(\left[\alpha_{n-1}\right]\right)$ comes from obstructions like $o_{n, n-1}^{q}\left(\left[\alpha^{\prime}\right]\right)$. Therefore we can get the following corollary immediately from Proposition 2.8 and Proposition 2.9.

Corollary 2.10. Let $[\beta]$ be an nontrivial element of $H^{q+1}\left(E_{0}^{\cdot}\right)$. Then $[\beta]$ is a second class obstructed element if and only if there exist $n \geq 0$ and $\alpha_{n-1}$ in $H^{q}\left(\mathscr{E}_{0} \otimes_{\mathscr{O}_{B, 0}} \mathcal{O}_{B, 0} / m_{0}^{n}\right)$ such that $o_{n, n-1}^{q}\left(\left[\alpha_{n-1}\right]\right)=\rho_{n-1}^{q+1}([\beta])$.

Let us come back to our problem. Suppose $\alpha$ can be extended to an element $\alpha_{n-1}$ such that $j_{0}^{n-1}\left(d^{q}\left(\alpha_{n-1}\right)\right)(t)=0$. What we care is whether $\alpha$ can be extended to an element which belongs to $\operatorname{Ker}\left(d^{q}\right)$ in a neighborhood of 0 , so if we have an $n$-th order extension $\alpha_{n}$ of $\alpha$, it is not necessary that $j_{0}^{i-1}\left(\alpha_{n}-\alpha_{n-1}\right)(t)=0$, for 
all $1<i<n$. What we need is just $j_{0}^{0}\left(\alpha_{n}-\alpha_{n-1}\right)(t)=0$ which means $\alpha_{n}$ is an extension of $\alpha$. So the "real" obstructions come from $o_{n, n-1}^{q}\left(\left[\alpha_{n-1}\right]\right)$. The relation between the obstructions $o_{n, n-1}^{q}\left(\left[\alpha_{n-1}\right]\right)$ and the jumping phenomenon of Hodge numbers is the following.

Theorem 2.11. Let $\pi: \mathscr{X} \rightarrow B$ be a small deformation of the central fiber compact complex manifold $X$. Now we consider $\operatorname{dim} H^{q}\left(X_{t}, \Omega_{X_{t}}^{p}\right)$ as a function of $t \in B$. It jumps at $t=0$ if and only if there exists an element $\alpha$ either in $H^{q}\left(X, \Omega_{X}^{p}\right)$ or in $H^{q-1}\left(X, \Omega_{X}^{p}\right)$ and a minimal natural number $n \geq 1$ such that the $n$-th order obstruction

$$
o_{n, n-1}(\alpha) \neq 0 \text {. }
$$

Proof. By the Grauert's Direct Image Theorem, for the given $p$, there exists a complex of vector bundles $\left(E^{\cdot}, d^{*}\right)$, such that for arbitrary $t \in B$,

$$
H^{q}\left(X_{t}, \Omega_{X_{t}}^{p}\right)=H^{q}\left(E_{t}^{\cdot}\right)=\operatorname{Ker}\left(d^{q}\right) / \operatorname{Im}\left(d^{q-1}\right) .
$$

Therefore, $\operatorname{dim} H^{q}\left(X_{t}, \Omega_{X_{t}}^{p}\right)$ jumps at $t=0$ if and only if $\operatorname{dim} H^{q}\left(E_{t}^{*}\right)$ jumps at $t=0$. While $\operatorname{dim} H^{q}\left(E_{t}^{*}\right)$ jumps at $t=0$ if and only if either $\operatorname{dim} \operatorname{Ker}\left(d^{q}\right)$ or $\left(-\operatorname{dim} \operatorname{Im}\left(d^{q-1}\right)\right)$ jumps at $t=0$. So $\operatorname{dim} H^{q}\left(X_{t}, \Omega_{X_{t}}^{p}\right)$ jumps at $t=0$ if and only if either $\operatorname{dim} \operatorname{Ker}\left(d^{q}\right)$ or $\operatorname{dim} \operatorname{Ker}\left(d^{q-1}\right)$ jumps at $t=0$.

So we can see that if $\operatorname{dim} H^{q}\left(X_{t}, \Omega_{X_{t}}^{p}\right)$ does not jump at $t=0$ then both $\operatorname{Ker}\left(d^{q}\right)$ and $\operatorname{Ker}\left(d^{q-1}\right)$ are holomorphic subbundles. And so for arbitrary element $\alpha$ in $\left.\operatorname{Ker}\left(d^{q}\right)\right|_{t=0}$ or in $\left.\operatorname{Ker}\left(d^{q-1}\right)\right|_{t=0}, \alpha$ can be extended to a section of $\operatorname{Ker}\left(d^{q}\right)$ or $\operatorname{Ker}\left(d^{q-1}\right)$. Combine with Proposition 2.6, we get the proof of the sufficiency of the statement.

For the necessity, I need the claim below. With this claim we get the necessity of the statement immediately.

Claim. For a given $q$, $\operatorname{dim} \operatorname{Ker}\left(d^{q}\right)$ jumps at $t=0$ if and only if there exists an element $\left.\alpha \in \operatorname{Ker}\left(d^{q}\right)\right|_{t=0}$ and $n \geq 1$ such that

$$
o_{n, n-1}(\alpha) \neq 0 \text {. }
$$

Proof of the claim. First I want to prove the claim for the case when $E^{q}$ is a line bundle. Via a local trivialization of $E^{q}, d^{q}$ is represented by a holomorphic function $d^{q}(t)$. Let $n$ be the order of $d^{q}(t)$ at $t=0$; then for arbitrary nontrivial $\left.\alpha \in \operatorname{Ker}\left(d^{q}\right)\right|_{t=0}, \alpha$ cannot be extended to an $n$-th order extension $\alpha_{n}$ (that is, $\alpha_{n}$ is a section of $E^{q}$ such that $\alpha_{n}(0)=\alpha$ and $d^{q}\left(\alpha_{n}\right)=0$ up to order $\left.n\right)$. Together with Proposition 2.6 we get the proof of the claim in this case.

In the general case, let $K$ be the biggest locally free subsheaf of $\operatorname{Ker}\left(d^{q}\right)$; then we get a quotient bundle $E^{q} / K$. Let $r$ be the rank of $E^{q} / K$. Then $d^{q}$ induces a 
map

$$
\operatorname{det}\left(d^{q}\right): \bigwedge^{r}\left(E^{q} / K\right) \rightarrow \bigwedge^{r}\left(E^{q+1}\right) .
$$

For arbitrary $\left.\alpha \in \operatorname{Ker}\left(d^{q}\right)\right|_{t=0}$, let $\alpha^{\prime}$ be an extension of $\alpha$. Denote by $\alpha^{\prime \prime}$ the image of $\alpha^{\prime}$ in $E^{q} / K$. Let $e_{1}, \ldots, e_{r-1}, \alpha^{\prime \prime}(0)$ be a basis of $E^{q} /\left.K\right|_{t=0}$ and let $e_{1}^{\prime}, \ldots, e_{r-1}^{\prime}$ be an extension of $e_{1}, \ldots, e_{r-1}$. Then we get a section $\alpha^{\prime \prime} \wedge e_{1}^{\prime} \wedge \cdots \wedge e_{r-1}^{\prime}$ of $\wedge^{r}\left(E^{q} / K\right)$. It is not difficult to see that if $\alpha^{\prime}$ is an $n$-th order extension of $\alpha$, then $\alpha^{\prime \prime} \wedge e_{1}^{\prime} \wedge \cdots \wedge e_{r-1}^{\prime}$ is an extension of $\alpha^{\prime \prime}(0) \wedge e_{1} \wedge \cdots \wedge e_{r-1}$. And the order of $\alpha^{\prime \prime} \wedge e_{1}^{\prime} \wedge \cdots \wedge e_{r-1}^{\prime}$ is at least $n$, which means $\operatorname{det}\left(d^{q}\right)\left(\alpha^{\prime \prime} \wedge e_{1}^{\prime} \wedge \cdots \wedge e_{r-1}^{\prime}\right)$ is equal to 0 up to order $n$. From the proof of the case of line bundle, we know that there exists $n$ such that for arbitrary nontrivial $\left.\beta \in \bigwedge^{r}\left(E^{q} / K\right)\right|_{t=0}$, we cannot extend $\beta$ to an $n$-th order extension. So for arbitrary $\left.\alpha \in \operatorname{Ker}\left(d^{q}\right)\right|_{t=0}$, we cannot get an extension of $\alpha$ with order more than $n-1$. Together with Proposition 2.6, we get the proof of the general case.

Since these obstructions are so important when we consider the problem of variation of Hodge numbers, we will try to find out an explicit calculation for such obstructions in the next section.

\section{The formula for the obstructions}

We are going to prove in this section an explicit formula (Theorem 3.5) for the abstract obstructions described above. Let $\pi: \mathscr{L} \rightarrow B$ be a deformation of $\pi^{-1}(0)=$ $X$, where $X$ is a compact complex manifold. For every integer $n \geq 0$, denote by $B_{n}=$ Spec $\mathcal{O}_{B, 0} / m_{0}^{n+1}$ the $n$-th order infinitesimal neighborhood of the closed point $0 \in B$ of the base $B$. Let $X_{n} \subset \mathscr{X}$ be the complex space over $B_{n}$. Let $\pi_{n}: X_{n} \rightarrow B_{n}$ be the $n$-th order deformation of $X$. In order to study the jumping phenomenon of Dolbeault cohomology groups, for arbitrary $[\alpha]$ that belongs to $H^{q}\left(X, \Omega_{X}^{p}\right)$, suppose we can extend $[\alpha]$ to order $n-1$ in $H^{q}\left(X_{n-1}, \Omega_{X_{n-1} / B_{n-1}}^{p}\right)$. Denote such elements by $\left[\alpha_{n-1}\right]$. In the following, we try to find out the obstruction of the extension of $\left[\alpha_{n-1}\right]$ to $n$-th order. Denote $\pi^{*}\left(m_{0}\right)$ by $\mu_{0}$. Consider the exact sequence

$$
0 \rightarrow M_{0}^{n} / M_{0}^{n+1} \otimes \Omega_{X_{0} / B_{0}}^{p} \rightarrow \Omega_{X_{n} / B_{n}}^{p} \rightarrow \Omega_{X_{n-1} / B_{n-1}}^{p} \rightarrow 0
$$

which induces a long exact sequence

$$
\begin{aligned}
0 \rightarrow H^{0}\left(X, \mu_{0}^{n} / M_{0}^{n+1} \otimes \Omega_{X_{0} / B_{0}}^{p}\right) \rightarrow H^{0}\left(X_{n}, \Omega_{X_{n} / B_{n}}^{p}\right) & \\
& \rightarrow H^{0}\left(X_{n-1}, \Omega_{X_{n-1} / B_{n-1}}^{p}\right) \rightarrow H^{1}\left(X, \mu_{0}^{n} / M_{0}^{n+1} \otimes \Omega_{X_{0} / B_{0}}^{p}\right) \rightarrow \cdots
\end{aligned}
$$

The obstruction for $\left[\alpha_{n-1}\right]$ comes from the nontrivial image of the connecting homomorphism 


$$
\delta^{*}: H^{q}\left(X_{n-1}, \Omega_{X_{n-1} / B_{n-1}}^{p}\right) \rightarrow H^{q+1}\left(X, \mu_{0}^{n} / M_{0}^{n+1} \otimes \Omega_{X_{0} / B_{0}}^{p}\right) .
$$

We will calculate it by $\check{C}$ ech calculation.

Cover $X$ by open sets $U_{i}$ such that, for arbitrary $i, U_{i}$ is small enough. More precisely, $U_{i}$ is Stein and the exact sequence

$$
0 \rightarrow \pi_{n}^{*}\left(\Omega_{B_{n}}\right)\left(U_{i}\right) \rightarrow \Omega_{X_{n}}\left(U_{i}\right) \rightarrow \Omega_{X_{n} / B_{n}}\left(U_{i}\right) \rightarrow 0
$$

splits. So we have a map $\varphi_{i}: \Omega_{X_{n} / B_{n}}\left(U_{i}\right) \rightarrow \Omega_{X_{n}}\left(U_{i}\right)$ such that

$$
\varphi_{i}\left(\Omega_{X_{n} / B_{n}}\left(U_{i}\right)\right) \oplus \pi_{n}^{*}\left(\Omega_{B_{n}}\right)\left(U_{i}\right) \cong \Omega_{X_{n}}\left(U_{i}\right) .
$$

This decomposition determines a local decomposition of the exterior differentiation $d_{X_{n}}$ in $\Omega_{X_{n}}$ on each $U_{i}$

$$
d_{X_{n}}=d_{B_{n}}^{i}+d_{X_{n} / B_{n}}^{i} .
$$

By definition, $d_{X_{n} / B_{n}}$ is given by $\varphi_{i}^{-1} \circ d_{X_{n} / B_{n}}^{i} \circ \varphi_{i}$.

For a fixed open cover $\mathbf{U}$ of $\mathrm{X}$, denote the set of alternating $q$-cochains $\beta$ with values in $\mathscr{F}$ by $\mathscr{C}^{q}(\mathbf{U}, \mathscr{F})$, that is, to each $(q+1)$-tuple, $i_{0}<i_{1}<\cdots<i_{q}, \beta$ assigns a section $\beta\left(i_{0}, i_{1}, \ldots, i_{q}\right)$ of $\mathscr{F}$ over $U_{i_{0}} \cap U_{i_{1}} \cap \cdots \cap U_{i_{q}}$.

Let us still use $\varphi_{i}$ to denote the map

$$
\begin{gathered}
\varphi_{i}: \pi_{n}^{*}\left(\Omega_{B_{n}}^{r}\right) \wedge \Omega_{X_{n} / B_{n}}^{p}\left(U_{i}\right) \rightarrow \Omega_{X_{n}}^{p+r}\left(U_{i}\right), \\
\varphi_{i}\left(\omega_{i_{1}} \wedge \cdots \wedge \omega_{i_{r}} \wedge \beta_{j_{1}} \wedge \cdots \wedge \beta_{j_{p}}\right)=\omega_{i_{1}} \wedge \cdots \wedge \omega_{i_{r}} \wedge \varphi_{i}\left(\beta_{j_{1}}\right) \wedge \cdots \wedge \varphi_{i}\left(\beta_{j_{p}}\right) .
\end{gathered}
$$

Define

$$
\begin{gathered}
\varphi: \mathscr{C}^{q}\left(\mathbf{U}, \pi_{n}^{*}\left(\Omega_{B_{n}}^{r}\right) \wedge \Omega_{X_{n} / B_{n}}^{p}\right) \rightarrow \mathscr{C}^{q}\left(\mathbf{U}, \Omega_{X_{n}}^{p+r}\right), \\
\varphi(\beta)\left(i_{0}, i_{1}, \ldots, i_{q}\right)=\varphi_{i_{0}}\left(\beta\left(i_{0}, i_{1}, \ldots, i_{q}\right)\right)
\end{gathered}
$$

for all $\beta \in \mathscr{C}^{q}\left(\mathbf{U}, \pi_{n}^{*}\left(\Omega_{B_{n}}^{r}\right) \wedge \Omega_{X_{n} / B_{n}}^{p}\right)$, where $i_{0}<i_{1} \cdots<i_{q}$.

Define the total Lie derivative with respect to $B_{n}$,

$$
\begin{gathered}
L_{B_{n}}: \mathscr{C}^{q}\left(\mathbf{U}, \Omega_{X_{n}}^{p}\right) \rightarrow \mathscr{C}^{q}\left(\mathbf{U}, \Omega_{X_{n}}^{p+1}\right), \\
L_{B_{n}}(\beta)\left(i_{0}, i_{1}, \ldots, i_{q}\right)=d_{B_{n}}^{i_{0}}\left(\beta\left(i_{0}, i_{1}, \ldots, i_{q}\right)\right)
\end{gathered}
$$

for all $\beta \in \mathscr{C}^{q}\left(\mathbf{U}, \Omega_{X_{n}}^{p}\right)$, where $i_{0}<i_{1} \cdots<i_{q}$; see [Katz and Oda 1968].

Define, for each $U_{i}$, the total interior product with respect to $B_{n}$,

$$
I^{i}: \Omega_{X_{n}}^{p}\left(U_{i}\right) \rightarrow \Omega_{X_{n}}^{p}\left(U_{i}\right)
$$

by setting

$$
I^{i}\left(\mu d g_{1} \wedge d g_{2} \wedge \cdots \wedge d g_{p}\right)=\mu \sum_{j=1}^{p} d g_{1} \wedge \cdots \wedge d g_{j-1} \wedge d_{B_{n}}^{i}\left(g_{j}\right) \wedge d g_{j+1} \wedge \cdots \wedge d g_{p}
$$


When $p=0$, we put $I^{i}=0$; see [Katz and Oda 1968].

Define

$$
\begin{gathered}
\lambda: \mathscr{C}^{q}\left(\mathbf{U}, \Omega_{X_{n}}^{p}\right) \rightarrow \mathscr{C}^{q+1}\left(\mathbf{U}, \Omega_{X_{n}}^{p}\right), \\
(\lambda \beta)\left(i_{0}, \ldots, i_{q+1}\right)=\left(I^{i_{0}}-I^{i_{1}}\right) \beta\left(i_{1}, \ldots, i_{q+1}\right) \quad \forall \beta \in \mathscr{C}^{q}\left(\mathbf{U}, \Omega_{X_{n}}^{p}\right) .
\end{gathered}
$$

Lemma 3.1. $\lambda \circ \varphi=\delta \circ \varphi-\varphi \circ \delta \bmod \pi_{n}^{*}\left(\Omega_{B_{n}}^{2}\right) \wedge \Omega_{X_{n}}^{p-2}$.

Proof. Define

$$
\begin{gathered}
J: \mathscr{C}^{q}\left(\mathbf{U}, \Omega_{X_{n} / B_{n}}^{p}\right) \rightarrow \mathscr{C}^{q+1}\left(\mathbf{U}, \Omega_{X_{n}}^{p}\right), \\
(J(\beta))\left(i_{0}, \ldots, i_{q+1}\right)=(-1)\left(\varphi_{i_{0}}-\varphi_{i_{1}}\right)\left(\beta\left(i_{1}, \ldots, i_{q+1}\right)\right),
\end{gathered}
$$

where $i_{0}<i_{1}<\cdots<i_{q+1}$. For arbitrary $\beta$ belonging to $\mathscr{C}^{q}\left(\mathbf{U}, \Omega_{X_{n} / B_{n}}^{p}\right)$, $(\delta \circ \varphi(\beta))\left(i_{0}, \ldots, i_{q+1}\right)$

$$
\begin{aligned}
& =\sum_{j=0}^{q+1}(-1)^{j} \varphi(\beta)\left(i_{0}, \ldots, \widehat{i_{j}}, \ldots, i_{q+1}\right) \\
& =\varphi_{i_{1}}(\beta)\left(i_{1}, \ldots, i_{q+1}\right)+\sum_{j=1}^{q+1}(-1)^{j} \varphi_{i_{0}}(\beta)\left(i_{0}, \ldots, \widehat{i_{j}}, \ldots, i_{q+1}\right),
\end{aligned}
$$

while

$(\varphi \circ \delta(\beta))\left(i_{0}, \ldots, i_{q+1}\right)=$

$$
\varphi\left(\sum_{j=0}^{q+1}(-1)^{j} \beta\left(i_{0}, \ldots, \widehat{i_{j}}, \ldots, i_{q+1}\right)\right)=\sum_{j=0}^{q+1}(-1)^{j} \varphi_{i_{0}}(\beta)\left(i_{0}, \ldots, \widehat{i_{j}}, \ldots, i_{q+1}\right) .
$$

So we have $\delta \circ \varphi-\varphi \circ \delta=J$.

Fix $\left(i_{0}, \ldots, i_{q+1}\right)$ and let $\omega=\beta\left(i_{1}, \ldots, i_{q+1}\right)$. We must show that

$$
\left(I^{i_{0}}-I^{i_{1}}\right)\left(\varphi_{i_{1}}(\omega)\right)=-\left(\varphi_{i_{0}}-\varphi_{i_{1}}\right)(w) \bmod \pi_{n}^{*}\left(\Omega_{B_{n}}^{2}\right) \wedge \Omega_{X_{n}}^{p-2} .
$$

By linearity, we may suppose $\varphi_{i_{1}}(\omega)=\mu d g_{1} \wedge \cdots \wedge d g_{p}$. Then

$$
\begin{aligned}
\varphi_{i_{0}}(\omega) & =\mu d_{X_{n} / B_{n}}^{i_{0}}\left(g_{1}\right) \wedge \cdots \wedge d_{X_{n} / B_{n}}^{i_{0}}\left(g_{p}\right) \\
& =\mu\left(d g_{1}-d_{B_{n}}^{i_{0}}\left(g_{1}\right)\right) \wedge \cdots \wedge\left(d g_{p}-d_{B_{n}}^{i_{0}}\left(g_{p}\right)\right) \\
& =\mu d g_{1} \wedge \cdots \wedge d g_{p}-\sum_{j=1}^{p} \mu d g_{1} \wedge \cdots \wedge d g_{j-1} \wedge d_{B_{n}}^{i_{0}}\left(g_{j}\right) \wedge d g_{j+1} \wedge \cdots \wedge d g_{p} \\
& + \text { terms in } \pi_{n}^{*}\left(\Omega_{B_{n}}^{2}\right) \wedge \Omega_{X_{n}}^{p-2} .
\end{aligned}
$$

Thus

$$
\varphi_{i_{0}}(\omega)=\varphi_{i_{1}}(\omega)-I^{i_{0}} \circ \varphi_{i_{1}}(\omega) \bmod \pi_{n}^{*}\left(\Omega_{B_{n}}^{2}\right) \wedge \Omega_{X_{n}}^{p-2},
$$


and $I^{i_{1}} \circ \varphi_{i_{1}}=0$, which means

$$
\lambda \circ \varphi=J \quad \bmod \pi_{n}^{*}\left(\Omega_{B_{n}}^{2}\right) \wedge \Omega_{X_{n}}^{p-2} .
$$

Now we are ready to calculate the formula for the obstructions. Let

$$
\tilde{\alpha} \in \mathscr{C}^{q}\left(\mathbf{U}, \Omega_{X_{n} / B_{n}}^{p}\right)
$$

such that its quotient image in $\mathscr{b}^{q}\left(\mathbf{U}, \Omega_{X_{n-1} / B_{n-1}}^{p}\right)$ is $\alpha_{n-1}$. Then

$$
\delta^{*}\left(\left[\alpha_{n-1}\right]\right)=[\delta(\tilde{\alpha})],
$$

which is an element of

$$
H^{q+1}\left(X, M_{0}^{n} / M_{0}^{n+1} \otimes \Omega_{X_{0} / B_{0}}^{p}\right) \cong \mathrm{m}_{0}^{n} / \mathrm{m}_{0}^{n+1} \otimes H^{q+1}\left(X, \Omega_{X_{0} / B_{0}}^{p}\right) .
$$

Denote $r_{X_{n}}$ the restriction to the complex space $X_{n}$. In order to calculate the obstructions explicitly, we need to consider the map

$$
\rho: H^{q}\left(X, M_{0}^{n} / M_{0}^{n+1} \otimes \Omega_{X_{0} / B_{0}}^{p}\right) \rightarrow H^{q}\left(X_{n-1}, \pi_{n-1}^{*}\left(\Omega_{B_{n} \mid B_{n-1}}\right) \wedge \Omega_{X_{n-1} / B_{n-1}}^{p}\right)
$$

which is defined by $\rho[\sigma]=\left[\varphi^{-1} \circ r_{X_{n-1}} \circ L_{B_{n}} \circ \varphi(\sigma)\right]$.

\section{Lemma 3.2. The map}

$$
\rho: H^{q}\left(X, \mu_{0}^{n} / M_{0}^{n+1} \otimes \Omega_{X_{0} / B_{0}}^{p}\right) \rightarrow H^{q}\left(X_{n-1}, \pi_{n-1}^{*}\left(\Omega_{B_{n} \mid B_{n-1}}\right) \wedge \Omega_{X_{n-1} / B_{n-1}}^{p}\right)
$$

is well defined.

Proof. First, we need to show that if $\sigma$ is closed, then

$$
\varphi^{-1} \circ r_{X_{n-1}} \circ L_{B_{n}} \circ \varphi(\sigma)
$$

is closed, which is equivalent to show that

$$
\delta \circ r_{X_{n-1}} \circ L_{B_{n}} \circ \varphi(\sigma)=0 \bmod \pi_{n-1}^{*}\left(\Omega_{B_{n} \mid B_{n-1}}^{2}\right) \wedge \Omega_{X_{n} \mid X_{n-1}}^{p-1} .
$$

Note that $d_{X_{n}} \circ \delta=-\delta \circ d_{X_{n}}$. Then

$$
\begin{aligned}
\delta \circ r_{X_{n-1}} \circ L_{B_{n}} \circ \varphi(\sigma) & =r_{X_{n-1}} \circ \delta \circ L_{B_{n}} \circ \varphi(\sigma) \\
& =-r_{X_{n-1}} \circ\left(\delta \circ d_{X_{n} / B_{n}}+d_{X_{n} / B_{n}} \circ \delta+L_{B_{n}} \circ \delta\right) \circ \varphi(\sigma) .
\end{aligned}
$$

Since

$$
L_{B_{n}} \circ \delta \circ \varphi(\sigma) \equiv L_{B_{n}} \circ(\delta \circ \varphi-\lambda \circ \varphi)(\sigma) \equiv L_{B_{n}} \circ \varphi \circ \delta(\sigma)=0
$$

and

$$
r_{X_{n-1}} \circ\left(\delta \circ d_{X_{n} / B_{n}}+d_{X_{n} / B_{n}} \circ \delta\right) \circ \varphi(\sigma)=0,
$$

we have

$$
\delta \circ r_{X_{n-1}} \circ L_{B_{n}} \circ \varphi(\sigma) \equiv 0 \quad \bmod \pi_{n-1}^{*}\left(\Omega_{B_{n} \mid B_{n-1}}^{2}\right) \wedge \Omega_{X_{n} \mid X_{n-1}}^{p-1} .
$$


Next we need to show that if

$$
\sigma \in \mathscr{C}^{q}\left(\mathbf{U}, M_{0}^{n} / M_{0}^{n+1} \otimes \Omega_{X_{0} / B_{0}}^{p}\right),
$$

then $\varphi^{-1} \circ r_{X_{n-1}} \circ L_{B_{n}} \circ \varphi \circ \delta(\sigma)$ is exact. In fact, according to the calculation above,

$$
\begin{aligned}
r_{X_{n-1}} \circ L_{B_{n}} \circ \varphi \circ \delta(\sigma) & =-r_{X_{n-1}} \circ\left(\delta \circ d_{X_{n} / B_{n}}+d_{X_{n} / B_{n}} \circ \delta+\delta \circ L_{B_{n}}\right) \circ \varphi(\sigma) \\
& =-\delta \circ r_{X_{n-1}} \circ L_{B_{n}} \circ \varphi(\sigma) .
\end{aligned}
$$

In general, the map $\rho$ is not injective. However, as we mentioned at the end of the previous section, the "real" obstructions are not $o_{n}^{q}\left(\left[\alpha_{n-1}\right]\right)$ but $o_{n, n-1}^{q}\left(\left[\alpha_{n-1}\right]\right)$. So we don't need $\rho$ to be injective. In the following, we will explain that $\rho([\delta(\tilde{\alpha})])$ is exactly the "real" obstructions we need.

Lemma 3.3. For a certain direction, the action of $\rho([\delta(\tilde{\alpha})])$ is exactly

$$
o_{n, n-1}^{q}\left(\left[\alpha_{n-1}\right]\right)
$$

in the previous section.

Proof. In fact,

$$
\begin{aligned}
H^{q}\left(X_{n-1}, \pi_{n-1}^{*}\left(\Omega_{B_{n} \mid B_{n-1}}\right) \wedge \Omega_{X_{n-1} / B_{n-1}}^{p}\right) & \\
= & \left(\Omega_{B_{n} \mid B_{n-1}}\right) \otimes_{\mathbb{O}_{B_{n-1}}} H^{q}\left(X_{n-1}, \Omega_{X_{n-1} / B_{n-1}}^{p}\right) .
\end{aligned}
$$

Let $b=\operatorname{dim} B, t_{i}$ the local coordinates of $B$ for $i=0, \ldots, b$. Then $\rho([\delta(\tilde{\alpha})])$ can be written as $\sum_{i=0}^{b} d t_{i} \otimes \tilde{\alpha}_{i}$, where $\tilde{\alpha}_{i} \in H^{q}\left(X_{n-1}, \Omega_{X_{n-1} / B_{n-1}}^{p}\right)$. For a certain direction $\partial / \partial t_{i}$, suppose $\tilde{\alpha}_{i} \neq 0$. Then by a simple calculation, it is not difficult to check that $\tilde{\alpha}_{i}=$ constant $\cdot\left[\delta(\tilde{\alpha}) / t_{i}\right]$ in $H^{q}\left(X_{n-1}, \Omega_{X_{n-1} / B_{n-1}}^{p}\right)$. Note that $\left[\delta(\tilde{\alpha}) / t_{i}\right]$ is exactly the obstruction $o_{n, n-1}^{q}\left(\left[\alpha_{n-1}\right]\right)$ in the direction of $\partial / \partial t_{i}$ we mentioned in the previous section.

Now consider the exact sequence

$$
0 \rightarrow \pi_{n-1}^{*}\left(\Omega_{B_{n} \mid B_{n-1}}\right) \rightarrow \Omega_{X_{n} \mid X_{n-1}} \rightarrow \Omega_{X_{n-1} / B_{n-1}} \rightarrow 0 .
$$

The connecting homomorphism of the associated long exact sequence gives the Kodaira-Spencer class of order $n$ [Voisin 1996, 1.3.2]. By wedging the above exact sequence with $\Omega_{X_{n-1} / B_{n-1}}^{p-1}$, we get a new exact sequence. The connecting homomorphism of such an exact sequence gives us a map

$$
H^{q}\left(X_{n-1}, \Omega_{X_{n-1} / B_{n-1}}^{p}\right) \rightarrow H^{q+1}\left(X_{n-1}, \pi^{*}\left(\Omega_{B_{n} \mid B_{n-1}}\right) \wedge \Omega_{X_{n-1} / B_{n-1}}^{p-1}\right) .
$$

Denote this map by $\kappa_{n}\llcorner$, for this map is simply the interior product with the Kodaira-Spencer class of order $n$. By the definition and simple calculation, it is not difficult to prove the following lemma. 
Lemma 3.4. Let $[\theta]$ be an element of $H^{q}\left(X_{n-1}, \Omega_{X_{n-1} / B_{n-1}}^{p}\right)$, let $\tilde{\theta}$ be an element of $\mathscr{C}^{q}\left(\mathbf{U}, \Omega_{X_{n} / B_{n}}^{p}\right)$ such that its quotient image is $\theta$. Then $\left[\kappa_{n}\llcorner\theta]\right.$ is equal to $\left[\varphi^{-1} \circ\right.$ $\left.r_{X_{n-1}} \circ \delta \circ \varphi(\tilde{\theta})\right]$.

Proof. From the exact sequence (3-1), we get

$$
0 \rightarrow \pi_{n-1}^{*}\left(\Omega_{B_{n} \mid B_{n-1}}\right) \wedge \Omega_{X_{n-1} / B_{n-1}}^{p-1} \rightarrow \Omega_{X_{n} \mid X_{n-1}} \wedge \Omega_{X_{n-1} / B_{n-1}}^{p-1} \rightarrow \Omega_{X_{n-1} / B_{n-1}}^{p} \rightarrow 0 .
$$

Let

$$
[\theta] \in H^{q}\left(X_{n-1}, \Omega_{X_{n-1} / B_{n-1}}^{p}\right) .
$$

By the definition of Kodaira-Spencer class, $\delta^{*}[\theta]=\left[\kappa_{n}\llcorner\theta]\right.$. By the definition of $\delta^{*}$, if we can find an element $\hat{\theta}$ of

$$
\mathscr{C}^{q}\left(\mathbf{U}, \Omega_{X_{n} \mid X_{n-1}} \wedge \Omega_{X_{n-1} / B_{n-1}}^{p-1}\right)
$$

such that its quotient image is $\theta$, then $[\delta(\hat{\theta})]=\left[\kappa_{n}\llcorner\theta]\right.$. On the other hand,

$$
\Omega_{X_{n} \mid X_{n-1}} \wedge \Omega_{X_{n-1}^{p-1} B_{n-1}}^{p-1}
$$

is equal to the quotient of $\Omega_{X_{n} \mid X_{n-1}}^{p}$ by

$$
\pi_{n-1}^{*}\left(\Omega_{B_{n} \mid B_{n-1}}^{2}\right) \wedge \Omega_{X_{n} \mid X_{n-1}}^{p-2} .
$$

In order to calculate $\delta(\hat{\theta})$, we can take the preimage $\hat{\theta}^{\prime}$ of $\hat{\theta}$ in $\varphi^{q}\left(\mathbf{U}, \Omega_{X_{n} \mid X_{n-1}}^{p}\right)$. Then $\delta(\hat{\theta})$ is equal to the quotient image of $\delta\left(\hat{\theta}^{\prime}\right)$. By a simple calculation, the quotient of the quotient image of $r_{X_{n-1}} \circ \varphi(\tilde{\theta})$ is $\theta$. Therefore

$$
\left[\kappa_{n}\llcorner\theta]=\left[\varphi^{-1} \circ r_{X_{n-1}} \circ \delta \circ \varphi(\tilde{\theta})\right],\right.
$$

which gives us the proof of this lemma.

With the above preparation, we are ready to prove the main theorem of this section.

Theorem 3.5. Let $\pi: \mathscr{X} \rightarrow B$ be a deformation of $\pi^{-1}(0)=X$, where $X$ is a compact complex manifold. Let $\pi_{n}: X_{n} \rightarrow B_{n}$ be the $n$-th order deformation of $X$. For arbitrary $[\alpha]$ belongs to $H^{q}\left(X, \Omega^{p}\right)$, suppose we can extend $[\alpha]$ to order $n-1$ in $H^{q}\left(X_{n-1}, \Omega_{X_{n-1} / B_{n-1}}^{p}\right)$. Denote such elements by $\left[\alpha_{n-1}\right]$. The obstruction of the extension of $[\alpha]$ to the $n$-th order is

$$
o_{n, n-1}(\alpha)=d_{X_{n-1} / B_{n-1}} \circ \kappa_{n}\left\llcorner\left(\alpha_{n-1}\right)+\kappa_{n}\left\llcorner\circ d_{X_{n-1} / B_{n-1}}\left(\alpha_{n-1}\right),\right.\right.
$$

where $\kappa_{n}$ is the $n$-th order Kodaira-Spencer class and $d_{X_{n-1} / B_{n-1}}$ is the relative differential operator of $(n-1)$-th order deformation. 
Proof.

$$
\begin{aligned}
r_{X_{n-1}} \circ L_{B_{n}} \circ \varphi \circ \delta(\tilde{\alpha}) & \equiv r_{X_{n-1}} \circ L_{B_{n}} \circ(\delta \circ \varphi-\lambda \circ \varphi)(\tilde{\alpha}) \\
& \equiv r_{X_{n-1}} \circ L_{B_{n}} \circ \delta \circ \varphi(\tilde{\alpha}) \\
& \equiv-r_{X_{n-1}} \circ\left(d_{X_{n} / B_{n}} \circ \delta+\delta \circ d_{X_{n} / B_{n}}+\delta \circ L_{B_{n}}\right) \circ \varphi(\tilde{\alpha}) \\
& \equiv-r_{X_{n-1}} \circ\left(d_{X_{n} / B_{n}} \circ \delta+\delta \circ d_{X_{n} / B_{n}}\right) \circ \varphi(\tilde{\alpha})-\delta \circ r_{X_{n-1}} \circ L_{B_{n}} \circ \varphi(\tilde{\alpha}) .
\end{aligned}
$$

Therefore,

$$
\begin{aligned}
& {\left[r_{X_{n-1}} \circ L_{B_{n}} \circ \varphi \circ \delta(\tilde{\alpha})\right]} \\
& \quad=\left[-r_{X_{n-1}} \circ\left(d_{X_{n} / B_{n}} \circ \delta+\delta \circ d_{X_{n} / B_{n}}\right) \circ \varphi(\tilde{\alpha})\right] \\
& \quad=-\left[d_{X_{n-1} / B_{n-1}} \circ r_{X_{n-1}} \delta \circ \varphi(\tilde{\alpha})+r_{X_{n-1}} \circ \delta \circ d_{X_{n} / B_{n}} \circ \varphi(\tilde{\alpha})\right] \\
& \quad=-\left[d_{X_{n-1} / B_{n-1}} \circ \varphi \circ \varphi^{-1} \circ r_{X_{n-1}} \delta \circ \varphi(\tilde{\alpha})+r_{X_{n-1}} \circ \delta \circ \varphi \circ d_{X_{n} / B_{n}}(\tilde{\alpha})\right] \\
& \quad=-\left[\varphi \circ d_{X_{n-1} / B_{n-1}} \circ \varphi^{-1} \circ r_{X_{n-1}} \delta \circ \varphi(\tilde{\alpha})+r_{X_{n-1}} \circ \delta \circ \varphi \circ\left(d_{X_{n-1} / B_{n-1}}\left(\alpha_{n-1}\right)\right)\right] \\
& \quad=-\left[d _ { X _ { n - 1 } / B _ { n - 1 } } \circ \kappa _ { n } \left\llcorner\alpha_{n-1}+\kappa_{n}\left\llcorner\circ d_{X_{n-1} / B_{n-1}}\left(\alpha_{n-1}\right)\right]\right.\right.
\end{aligned}
$$

By this theorem, we can get the following corollary immediately.

Corollary 3.6. Let $\pi: \mathscr{L} \rightarrow B$ be a deformation of $\pi^{-1}(0)=X$, where $X$ is a compact complex manifold. Suppose that up to order $n$, the $d_{1}$ of the Frölicher spectral sequence vanishes. For arbitrary $[\alpha]$ that belongs to $H^{q}\left(X, \Omega_{X}^{p}\right)$, it can be extended to order $n+1$ in $H^{q}\left(X_{n+1}, \Omega_{X_{n+1} / B_{n+1}}^{p}\right)$.

Proof. This result can be shown by induction on $k$.

Suppose that the corollary is proved for $k-1$; then we can extend $[\alpha]$ to an element $\left[\alpha_{k-1}\right]$ in $H^{q}\left(X_{k-1}, \Omega_{X_{k-1} / B_{k-1}}^{p}\right)$. By Theorem 3.5, the obstruction for the extension of $[\alpha]$ to $k$-th order comes from

$$
o_{k, k-1}(\alpha)=d_{X_{k-1} / B_{k-1}} \circ \kappa_{k}\left\llcorner\left(\alpha_{k-1}\right)+\kappa_{k}\left\llcorner\circ d_{X_{k-1} / B_{k-1}}\left(\alpha_{k-1}\right) .\right.\right.
$$

By assumption,

$$
d_{X_{k-1} / B_{k-1}}: H^{q^{\prime}}\left(X_{k-1}, \Omega_{X_{k-1} / B_{k-1}}^{p^{\prime}}\right) \rightarrow H^{q^{\prime}}\left(X_{k-1}, \Omega_{X_{k-1} / B_{k-1}}^{p^{\prime}+1}\right)
$$

is trivial, where $k \leq n, 0 \leq q^{\prime} \leq \operatorname{dim} X, 0 \leq p^{\prime} \leq \operatorname{dim} X$. So we have

$$
d_{X_{k-1} / B_{k-1}}\left(\alpha_{k-1}\right)=0 \quad \text { and } \quad d_{X_{k-1} / B_{k-1}} \circ \kappa_{k}\left\llcorner\left(\alpha_{k-1}\right)=0 .\right.
$$

So the obstruction $o_{k, k-1}(\alpha)$ is trivial which means $[\alpha]$ can be extended to $k$-th order.

Combining Corollary 3.6 and Theorem 2.11, we get the following corollary. 
Corollary 3.7. Let $\pi: \mathscr{X} \rightarrow B$ be a deformation of $\pi^{-1}(0)=X$, where $X$ is a compact complex manifold. Suppose the $d_{1}$ of the Frölicher spectral sequence vanishes for any order. Then the Hodge numbers of $X$ are stable in this deformation.

\section{An example}

In this section, we will use the formula in the previous section to study the jumping phenomenon of the Hodge numbers $h^{p, q}$ of small deformations of Iwasawa manifold. It was Kodaira who first calculated small deformations of Iwasawa manifold [Nakamura 1975]. In the first part of this section, let us recall his result.

Set

$G=\left\{\left(\begin{array}{ccc}1 & z_{2} & z_{3} \\ 0 & 1 & z_{1} \\ 0 & 0 & 1\end{array}\right): z_{i} \in \mathbb{C}\right\} \cong \mathbb{C}^{3}, \quad \Gamma=\left\{\left(\begin{array}{ccc}1 & \omega_{2} & \omega_{3} \\ 0 & 1 & \omega_{1} \\ 0 & 0 & 1\end{array}\right): \omega_{i} \in \mathbb{Z}+\mathbb{Z} \sqrt{-1}\right\}$.

The multiplication is defined by

$$
\left(\begin{array}{ccc}
1 & z_{2} & z_{3} \\
0 & 1 & z_{1} \\
0 & 0 & 1
\end{array}\right)\left(\begin{array}{ccc}
1 & \omega_{2} & \omega_{3} \\
0 & 1 & \omega_{1} \\
0 & 0 & 1
\end{array}\right)=\left(\begin{array}{ccc}
1 & z_{2}+\omega_{2} & z_{3}+\omega_{1} z_{2}+\omega_{3} \\
0 & 1 & z_{1}+\omega_{1} \\
0 & 0 & 1
\end{array}\right) \text {. }
$$

$X=G / \Gamma$ is called Iwasawa manifold. We may consider $X=\mathbb{C}^{3} / \Gamma . g \in \Gamma$ acts on $\mathbb{C}^{3}$ as follows:

$$
z_{1}^{\prime}=z_{1}+\omega_{1}, \quad z_{2}^{\prime}=z_{2}+\omega_{2}, \quad z_{3}^{\prime}=z_{3}+\omega_{1} z_{2}+\omega_{3},
$$

where $g=\left(\omega_{1}, \omega_{2}, \omega_{3}\right)$ and $z^{\prime}=z \cdot g$. There exist holomorphic 1 -forms $\varphi_{1}, \varphi_{2}, \varphi_{3}$ which are linearly independent at every point on $X$ and are given by

$$
\varphi_{1}=d z_{1}, \quad \varphi_{2}=d z_{2}, \quad \varphi_{3}=d z_{3}-z_{1} d z_{2},
$$

so that

$$
d \varphi_{1}=d \varphi_{2}=0, \quad d \varphi_{3}=-\varphi_{1} \wedge \varphi_{2} .
$$

On the other hand we have holomorphic vector fields $\theta_{1}, \theta_{2}, \theta_{3}$ on $X$ given by

$$
\theta_{1}=\frac{\partial}{\partial z_{1}}, \quad \theta_{2}=\frac{\partial}{\partial z_{2}}+z_{1} \frac{\partial}{\partial z_{3}}, \quad \theta_{3}=\frac{\partial}{\partial z_{3}} .
$$

It is easy to see that

$$
\left[\theta_{1}, \theta_{2}\right]=-\left[\theta_{2}, \theta_{1}\right]=\theta_{3}, \quad\left[\theta_{1}, \theta_{3}\right]=\left[\theta_{2}, \theta_{3}\right]=0 .
$$

In view of [Nakamura 1975, Theorem 3], $H^{1}\left(X, \mathscr{O}_{X}\right)$ is spanned by $\bar{\varphi}_{1}, \bar{\varphi}_{2}$. Since the tangent sheaf of $X$ is isomorphic to $\mathcal{O}^{3}, H^{1}\left(X, T_{X}\right)$ is spanned by $\theta_{i} \bar{\varphi}_{\lambda}$ for $i=1,2,3$ and $\lambda=1,2$. 
Consider the small deformation of $X$ given by

$$
\psi(t)=\sum_{i=1}^{3} \sum_{\lambda=1}^{2} t_{i \lambda} \theta_{i} \bar{\varphi}_{\lambda} t-\left(t_{11} t_{22}-t_{21} t_{12}\right) \theta_{3} \bar{\varphi}_{3} t^{2} .
$$

We summarize the numerical characters of the deformations. The deformations are divided into the following three classes:

(i) $t_{11}=t_{12}=t_{21}=t_{22}=0 ; X_{t}$ is a parallelizable manifold.

(ii) $t_{11} t_{22}-t_{21} t_{12}=0$ and $\left(t_{11}, t_{12}, t_{21}, t_{22}\right) \neq(0,0,0,0) ; X_{t}$ is not parallelizable.

(iii) $t_{11} t_{22}-t_{21} t_{12} \neq 0 ; X_{t}$ is not parallelizable.

\begin{tabular}{c|ccccccccc} 
& $h^{1,0}$ & $h^{0,1}$ & $h^{2,0}$ & $h^{1,1}$ & $h^{0,2}$ & $h^{3,0}$ & $h^{2,1}$ & $h^{1,2}$ & $h^{0,3}$ \\
\hline (i) & 3 & 2 & 3 & 6 & 2 & 1 & 6 & 6 & 1 \\
(ii) & 2 & 2 & 2 & 5 & 2 & 1 & 5 & 5 & 1 \\
(iii) & 2 & 2 & 1 & 5 & 2 & 1 & 4 & 4 & 1
\end{tabular}

We now explain the jumping phenomenon of the Hodge numbers by using the obstruction formula. From [Cordero et al. 1999, Corollary 4.3], it follows that the Dolbeault cohomology groups are

$$
\begin{aligned}
H^{0}\left(X, \Omega_{X}\right) & =\operatorname{Span}_{\mathbb{C}}\left\{\left[\varphi_{1}\right],\left[\varphi_{2}\right],\left[\varphi_{3}\right]\right\}, \\
H^{1}\left(X, O_{X}\right) & =\operatorname{Span}_{\mathbb{C}}\left\{\left[\bar{\varphi}_{1}\right],\left[\bar{\varphi}_{2}\right]\right\}, \\
H^{0}\left(X, \Omega_{X}^{2}\right) & =\operatorname{Span}_{\mathbb{C}}\left\{\left[\varphi_{1} \wedge \varphi_{2}\right],\left[\varphi_{2} \wedge \varphi_{3}\right],\left[\varphi_{3} \wedge \varphi_{1}\right]\right\}, \\
H^{1}\left(X, \Omega_{X}\right) & =\operatorname{Span}_{\mathbb{C}}\left\{\left[\varphi_{i} \wedge \bar{\varphi}_{\lambda}\right]\right\}, i=1,2,3, \lambda=1,2, \\
H^{2}\left(X, O_{X}\right) & =\operatorname{Span}_{\mathbb{C}}\left\{\left[\bar{\varphi}_{2} \wedge \bar{\varphi}_{3}\right],\left[\bar{\varphi}_{3} \wedge \bar{\varphi}_{1}\right]\right\}, \\
H^{0}\left(X, \Omega_{X}^{3}\right) & =\operatorname{Span}_{\mathbb{C}}\left\{\left[\varphi_{1} \wedge \varphi_{2} \wedge \varphi_{3}\right]\right\}, \\
H^{1}\left(X, \Omega_{X}^{2}\right) & =\operatorname{Span}_{\mathbb{C}}\left\{\left[\varphi_{i} \wedge \varphi_{j} \wedge \bar{\varphi}_{\lambda}\right]\right\}, i, j=1,2,3, i<j, \lambda=1,2, \\
H^{2}\left(X, \Omega_{X}^{1}\right) & =\operatorname{Span}_{\mathbb{C}}\left\{\left[\varphi_{i} \wedge \bar{\varphi}_{2} \wedge \bar{\varphi}_{3}\right],\left[\varphi_{j} \wedge \bar{\varphi}_{1} \wedge \bar{\varphi}_{3}\right]\right\}, i, j=1,2,3, \\
H^{3}\left(X, O_{X}\right) & =\operatorname{Span}_{\mathbb{C}}\left\{\left[\bar{\varphi}_{1} \wedge \bar{\varphi}_{2} \wedge \bar{\varphi}_{3}\right]\right\} .
\end{aligned}
$$

For example, let us first consider $h^{2,0}$, in the class (ii) deformation. The KodairaSpencer class of the this deformation is

$$
\psi_{1}(t)=\sum_{i=1}^{3} \sum_{\lambda=1}^{2} t_{i \lambda} \theta_{i} \bar{\varphi}_{\lambda}, \quad \text { with } t_{11} t_{22}-t_{21} t_{12}=0 .
$$

It is easy to check that 


$$
\begin{aligned}
& o_{1}\left(\varphi_{1} \wedge \varphi_{2}\right)=\partial\left(\operatorname{int}\left(\psi_{1}(t)\right)\left(\varphi_{1} \wedge \varphi_{2}\right)\right)-\operatorname{int}\left(\psi_{1}(t)\right)\left(\partial\left(\varphi_{1} \wedge \varphi_{2}\right)\right)=0 \\
& o_{1}\left(t_{11} \varphi_{2} \wedge \varphi_{3}-t_{21} \varphi_{1} \wedge \varphi_{3}\right)=-\partial\left(\left(t_{11} t_{22}-t_{21} t_{12}\right) \varphi_{3} \wedge \bar{\varphi}_{2}\right)=0, \\
& o_{1}\left(\varphi_{2} \wedge \varphi_{3}\right)=t_{21} \varphi_{1} \wedge \varphi_{2} \wedge \bar{\varphi}_{1}+t_{22} \varphi_{1} \wedge \varphi_{2} \wedge \bar{\varphi}_{2} \\
& o_{1}\left(\varphi_{1} \wedge \varphi_{3}\right)=t_{11} \varphi_{1} \wedge \varphi_{2} \wedge \bar{\varphi}_{1}+t_{12} \varphi_{1} \wedge \varphi_{2} \wedge \bar{\varphi}_{2} .
\end{aligned}
$$

Therefore, we have shown that for an element of the subspace

$$
\operatorname{Span}_{\mathbb{C}}\left\{\left[\varphi_{1} \wedge \varphi_{2}\right],\left[t_{11} \varphi_{2} \wedge \varphi_{3}-t_{21} \varphi_{1} \wedge \varphi_{3}\right]\right\},
$$

the first order obstruction is trivial, while, since $\left(t_{11}, t_{12}, t_{21}, t_{22}\right) \neq(0,0,0,0)$, at least one of the obstructions $o_{1}\left(\varphi_{2} \wedge \varphi_{3}\right), o_{1}\left(\varphi_{1} \wedge \varphi_{3}\right)$ is nontrivial, which partly explain why the Hodge number $h^{2,0}$ jumps from 3 to 2 . For another example, let us consider $h^{1,2}$, in the class (ii) deformation. It is easy to check that for an element of the subspace (the dimension of such a subspace is 5)

$\operatorname{Span}_{\mathbb{C}}\left\{\left[\varphi_{i} \wedge \bar{\varphi}_{\lambda} \wedge \bar{\varphi}_{3}\right],\left[t_{12} \varphi_{3} \wedge \bar{\varphi}_{2} \wedge \bar{\varphi}_{3}-t_{11} \varphi_{3} \wedge \bar{\varphi}_{1} \wedge \bar{\varphi}_{3}\right]\right\}, i=1,2, \lambda=1,2$,

the first order obstruction is trivial, while at least one of the obstructions

$$
o_{1}\left(\varphi_{3} \wedge \bar{\varphi}_{2} \wedge \bar{\varphi}_{3}\right), o_{1}\left(\varphi_{3} \wedge \bar{\varphi}_{1} \wedge \bar{\varphi}_{3}\right)
$$

is nontrivial.

Remark 4.1. It is easy to see that, in the class (ii) or (iii) deformation, the first order obstruction for any element in $H^{1}\left(X, \Omega_{X}\right)$ is trivial. The reason of the jump of the Hodge number $h^{1,1}$ from 6 to 5 comes from the existence of the second class obstructed elements $o_{1}\left(\varphi_{3}\right)$. After simple calculation, it is not difficult to get the structure equation of $X_{t}$ for $t \neq 0$,

$$
\left\{\begin{array}{l}
d \varphi_{1}=0 \\
d \varphi_{2}=0 \\
d \varphi_{3}=-\varphi_{1} \wedge \varphi_{2}+t o_{1}\left(\varphi_{3}\right),
\end{array}\right.
$$

which can be considered as an example of Proposition 2.8.

From the example we discussed above, it is not difficult to find out the following fact.

Proposition 4.2. Let $X$ be an non-Kähler nilpotent complex parallelizable manifold whose dimension is more than 2 , and $\pi: \mathscr{X} \rightarrow B$ the versal deformation family of $X$. Then the Hodge number $h^{1,0}$ jumps in any neighborhood of $0 \in B$.

Proof. Let $\varphi_{i}, i=1, \ldots, n, n=\operatorname{dim} X$ be the linearly independent holomorphic 1forms of $X$. By [Nakamura 1975, Theorem 3], $H^{1}\left(X, O_{X}\right)$ is spanned by a subset of $\left\{\bar{\varphi}_{i}\right\}, i=1, \ldots, n$. So we have $\partial: H^{1}\left(X, O_{X}\right) \rightarrow H^{1}\left(X, \Omega_{X}\right)$ is trivial, which means one term of the first order obstruction of the holomorphic 1-forms vanishes. Let 
$\theta_{i}$ for $i=1, \ldots, n$ be the dual of $\varphi_{i}$, which are linearly independent holomorphic vector fields. Since $X$ is non-Kähler, which means $X$ is not a torus, there exist $\varphi_{i}$ such that $\partial \varphi_{i} \neq 0$. Since $X$ is nilpotent, there exist $\varphi_{j}$ such that $\partial \varphi_{j}=0$. Assume that $\left.\partial \varphi_{i}=A \varphi_{k} \wedge \varphi_{l}+\cdots\right)$ with $A \neq 0$. Consider $\theta_{k} \bar{\varphi}_{j}$ in $H^{1}\left(X, T_{X}\right)$. It is easy to check that $o_{1}\left(\varphi_{i}, \theta_{k} \bar{\varphi}_{j}\right) \neq 0$.

\section{Acknowledgments}

The research was partially supported by China-France-Russian mathematics collaboration grant, No.34000-3275100, from Sun Yat-sen University. The author is grateful to the École Normale Supérieure in Paris for its hospitality during the academic years of 2005-2007. The author also thanks the referee for a thorough reading and many constructive suggestions and comments, which made the paper more readable, and Professor Voisin for her patient help and valuable suggestions.

\section{References}

[Bell and Narasimhan 1990] S. R. Bell and R. Narasimhan, "Proper holomorphic mappings of complex spaces", pp. 1-38 in Several complex variables, VI, edited by W. Barth and R. Narasimhan, Encyclopaedia Math. Sci. 69, Springer, Berlin, 1990. MR 92m:32046 Zbl 0733.32021

[Cordero et al. 1999] L. A. Cordero, M. Fernandez, L. Ugarte, and A. Gray, "Frölicher spectral sequence of compact nilmanifolds with nilpotent complex structure", pp. 77-102 in New developments in differential geometry (Budapest 1996), edited by J. Szenthe, Kluwer Acad. Publ., Dordrecht, 1999. MR 2000a:53126 Zbl 0943.57015

[Iitaka 1972] S. Iitaka, "Genus and classification of algebraic varieties. I", Sûgaku 24:1 (1972), 1427. MR 58 \#27976

[Katz and Oda 1968] N. M. Katz and T. Oda, "On the differentiation of de Rham cohomology classes with respect to parameters", J. Math. Kyoto Univ. 8 (1968), 199-213. MR 38 \#5792 Zbl 0165.54802

[Nakamura 1975] I. Nakamura, "Complex parallelisable manifolds and their small deformations", J. Differential Geometry 10 (1975), 85-112. MR 52 \#14389 Zbl 0297.32019

[Voisin 1996] C. Voisin, Symétrie miroir, Panoramas et Synthèses 2, Société Mathématique de France, Paris, 1996. MR 97i:32026 Zbl 0849.14001

[Voisin 2002] C. Voisin, Hodge theory and complex algebraic geometry. I, Cambridge Studies in Advanced Mathematics 76, Cambridge University Press, Cambridge, 2002. Translated from the French original by Leila Schneps. MR 2004d:32020 Zbl 1005.14002

Received November 20, 2007.

XUANMing Ye

DEPARTMENT OF MATHEMATICS

SUN YAT-SEN UNIVERSITY

GUANGZHOU 510275

CHINA

xuanming.ye@ens.fr 